\title{
Erratum to: Ricci solitons and odd-dimensional spheres
}

\author{
Jong Taek Cho
}

Published online: 10 February 2010

(C) Springer-Verlag 2010

\section{Erratum to: Monatsh Math DOI 10.1007/s00605-009-0095-1}

In Remark 3.2 in [3] Perelman showed that a Ricci soliton on a compact manifold is always replaced by a gradient Ricci soliton. Indeed, on a compact Riemannian manifold $(M, g)$ a potential vector field $V$ is the sum of a Killing field and a gradient (cf. [2]).

Hence, Theorem 6 in [1] should be changed to:

Theorem 6 Let $M$ be a complete real hypersurface in $\mathbb{C}^{n}$. If $M$ admits a Ricci soliton whose potential vector field is the Reeb vector field, then $M$ is a Hopf hypersurface and is congruent to a sphere $S^{2 n-1}$, a hyperplane $\mathbb{R}^{2 n-1}$ or a cylinder over a complete plane curve $\gamma \times \mathbb{R}^{2 n-2}$.

Since the sphere is only a compact case among such spaces in Theorem 6, we have the following Theorem C (or Corollary 7).

Theorem C If a compact real hypersurface $M$ in a complex number space $\mathbb{C}^{n}$ admits a Ricci soliton whose potential vector field is the Reeb vector field, then $M$ is a sphere.

\section{References}

1. Cho, J.T.: Ricci solitons and odd-dimensional spheres. Monatsh. Math. doi:10.1007/s00605-009-0095-1

The online version of the original article can be found under doi:10.1007/s00605-009-0095-1.

J. T. Cho $(\bowtie)$

Department of Mathematics, Chonnam National University, CNU The Institute of Basic Sciences, Gwangju 500-757, Korea

e-mail: jtcho@chonnam.ac.kr 
2. Derdzinski, A.: Compact Ricci solitons (2010, preprint)

3. Perelman, G.: The entropy formula for the Ricci flow and its geometric applications. http://arXiv.org/ abs/math.DG/0211159 (2010, preprint) 